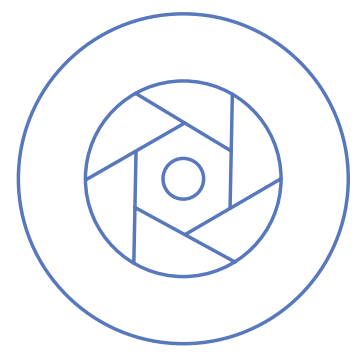

There is increasing epidemiological evidence linking early-life environmental exposures (i.e., maternal malnutrition/overnutrition, environmental chemicals, stress) with later-life health outcomese

Ann Nutr Metab 2020;76(suppl 3):4-14

\title{
Gestational Diabetes Mellitus and Developmental Programming
}

\section{Anne H.Y. Chu and Keith M. Godfrey}

Key insights

Gestational diabetes mellitus (GDM) affects an estimated 14\%

of pregnancies worldwide. It is now clear that children born

to mothers with GDM have an increased lifetime risk of

metabolic diseases compared to unexposed children. Other

long-term adverse consequences in the offspring include

cardiovascular abnormalities, dysregulation of glucose

metabolism, increased risk of allergic/respiratory disease, and

neurodevelopmental abnormalities. Together, these findings

highlight the importance of the intra-uterine environment as

a driver of epigenetic changes in the offspring.

\section{Current knowledge}

Several human studies have examined the association between in utero GDM exposure and DNA methylation in placentas, and offspring cord or infant blood. The findings have revealed several differentially methylated genes in the fetal tissues of babies born to mothers with GDM: of interest are those related to metabolic regulation, such as leptin, adiponectin, and the SLC2A1/GLUT1 and SLC2A3/GLUT3 genes. The effects of GDM, however, are not limited to offspring metabolism. Current research indicates that the epigenetic adaptations triggered by maternal glycemia also affect other developing organ systems in the infant, including neurodevelopment.

\section{Practical implications}

Infants born to mothers who receive GDM treatment (such as dietary advice, blood glucose monitoring, and insulin therapy) have improved perinatal outcomes. However, long-term fol-

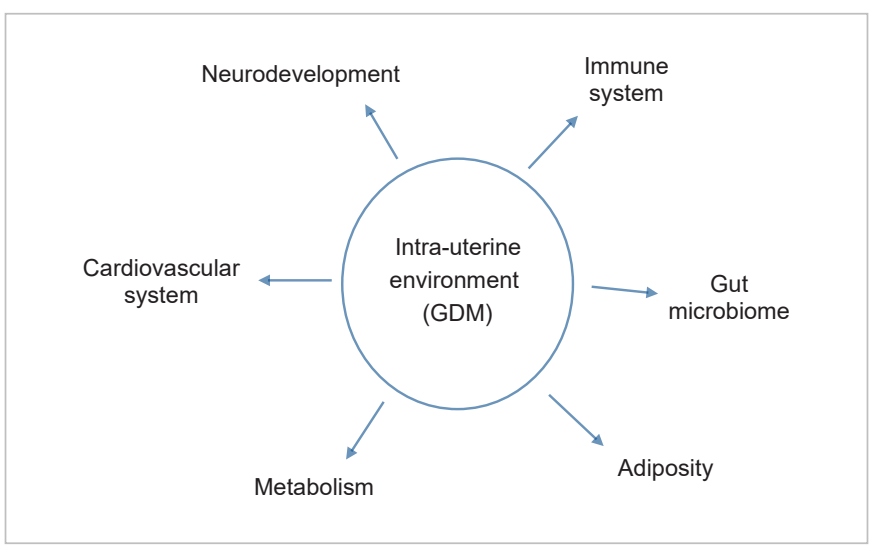

Gestational diabetes mellitus (GDM) affects many organ systems in the offspring through the mechanism of epigenetics.

low-up studies suggest that this treatment may not be sufficient to reduce childhood obesity in the offspring. The current evidence indicates that interventions delivered during pregnancy may only partly alter fetal growth and development, pointing towards the peri-conceptional period as an early modulator of health outcomes in the offspring. Further studies are needed to understand how we can leverage the peri-conceptional period as a window of opportunity for optimizing the health of future generations.

\section{Recommended reading}

Antoun E, Kitaba NT, Titcombe P, Dalrymple KV, Garratt ES, Barton SJ, et al. Maternal dysglycaemia, changes in the infant's epigenome modified with a diet and physical activity intervention in pregnancy: Secondary analysis of a randomised control trial. PLoS Med. 2020;17(11):e1003229. 